\title{
A prospective Phase II clinical trial of 5-aminolevulinic acid to assess the correlation of intraoperative fluorescence intensity and degree of histologic cellularity during resection of high-grade gliomas
}

\author{
Darryl Lau, MD,, Shawn L. Hervey-Jumper, MD, ${ }^{1}$ Susan Chang, MD, ${ }^{1}$ Annette M. Molinaro, PhD, ${ }^{1,2}$ \\ Michael W. McDermott, MD, ${ }^{1}$ Joanna J. Phillips, MD, PhD, ${ }^{1,3}$ and Mitchel S. Berger, MD1 \\ Departments of ${ }^{1}$ Neurological Surgery, ${ }^{2}$ Epidemiology and Biostatistics, and ${ }^{3}$ Pathology, University of California, \\ San Francisco, California
}

OBJECTIVE There is evidence that 5 -aminolevulinic acid (ALA) facilitates greater extent of resection and improves 6-month progression-free survival in patients with high-grade gliomas. But there remains a paucity of studies that have examined whether the intensity of ALA fluorescence correlates with tumor cellularity. Therefore, a Phase II clinical trial was undertaken to examine the correlation of intensity of ALA fluorescence with the degree of tumor cellularity.

METHODS A single-center, prospective, single-arm, open-label Phase II clinical trial of ALA fluorescence-guided resection of high-grade gliomas (Grade III and IV) was held over a 43-month period (August 2010 to February 2014). ALA was administered at a dose of $20 \mathrm{mg} / \mathrm{kg}$ body weight. Intraoperative biopsies from resection cavities were collected. The biopsies were graded on a 4-point scale (0 to 3 ) based on ALA fluorescence intensity by the surgeon and independently based on tumor cellularity by a neuropathologist. The primary outcome of interest was the correlation of ALA fluorescence intensity to tumor cellularity. The secondary outcome of interest was ALA adverse events. Sensitivities, specificities, positive predictive values (PPVs), negative predictive values (NPVs), and Spearman correlation coefficients were calculated.

RESULTS A total of 211 biopsies from 59 patients were included. Mean age was 53.3 years and $59.5 \%$ were male. The majority of biopsies were glioblastoma (GBM) (79.7\%). Slightly more than half (52.5\%) of all tumors were recurrent. ALA intensity of 3 correlated with presence of tumor $97.4 \%$ (PPV) of the time. However, absence of ALA fluorescence (intensity 0) correlated with the absence of tumor only $37.7 \%$ (NPV) of the time. For all tumor types, GBM, Grade III gliomas, and recurrent tumors, ALA intensity 3 correlated strongly with cellularity Grade 3; Spearman correlation coefficients (r) were $0.65,0.66,0.65$, and 0.62 , respectively. The specificity and PPV of ALA intensity 3 correlating with cellularity Grade 3 ranged from $95 \%$ to $100 \%$ and $86 \%$ to $100 \%$, respectively. In biopsies without tumor (cellularity Grade 0 ), $35.4 \%$ still demonstrated ALA fluorescence. Of those biopsies, 90.9\% contained abnormal brain tissue, characterized by reactive astrocytes, scattered atypical cells, or inflammation, and $8.1 \%$ had normal brain. In nonfluorescent (ALA intensity 0) biopsies, $62.3 \%$ had tumor cells present. The ALA-associated complication rate among the study cohort was $3.4 \%$.

CONCLUSIONS The PPV of utilizing the most robust ALA fluorescence intensity (lava-like orange) as a predictor of tumor presence is high. However, the NPV of utilizing the absence of fluorescence as an indicator of no tumor is poor. ALA intensity is a strong predictor for degree of tumor cellularity for the most fluorescent areas but less so for lower ALA intensities. Even in the absence of tumor cells, reactive changes may lead to ALA fluorescence.

http://thejns.org/doi/abs/10.3171/2015.5.JNS1577

KEY WORDS aminolevulinic acid; cellularity; fluorescence; glioblastoma; high-grade glioma; oncology

$\mathrm{O}$ VER the past several years, there has been an accumulation of studies that have shown that extent of resection (EOR) affects both overall- and progression-free survival in the surgical treatment of high-grade gliomas such as glioblastoma (GBM) ${ }^{1,3,12,14,24,33}$ Current evidence suggests that cytoreductive surgery is beneficial when at least $78 \%$ resection is obtained for high-grade gliomas. ${ }^{21}$ Thus, when possible and safe, it is considered important to achieve the greatest EOR while preserving neurological function.

ABBREVIATIONS ALA = 5-aminolevulinic acid; EOR = extent of resection; GBM = glioblastoma; GTR = gross-total resection; $\mathrm{H}$ \& $\mathrm{E}=$ hematoxylin and eosin; LFT = liver function test; NPV = negative predictive value; PPV = positive predictive value; $W H O=$ World Health Organization.

SUBMITTED January 21, 2015. ACCEPTED May 7, 2015.

INCLUDE WHEN CITING Published online November 6, 2015; DOI: 10.3171/2015.5.JNS1577. 
Resection of gliomas can be technically challenging as there is no definitive way to distinguish tumor from normal brain during the course of the procedure other than by gross visualization and tactile consistency. This is particularly true at the invasive tumor margin. Studies have shown that gross-total resection (GTR) of contrast-enhancing tumor is achieved in only about $20 \%-30 \%$ of patients, and in patients deemed to have tumors amendable to GTR, and radiographic complete resection is achieved in only approximately $24 \%$ of patients. ${ }^{1,3,16,24,33,35}$ These modest outcomes are likely due to difficulty in determining the distinction between tumor and brain parenchyma. ${ }^{1}$ Several technological advances have been explored to aid in improving the ability to obtain greater EOR during glioma surgery: intraoperative MRI,${ }^{15}$ neuronavigation, ${ }^{36}$ ultrasound, ${ }^{13}$ and newer technologies such as Raman scattered microscopy. ${ }^{10}$ The standard mode being used, with few exceptions, still relies on preoperative imaging studies and neuronavigation to guide the course of resection, as well as the use of clinical acumen.

Over the last several years, 5-aminolevulinic acid (ALA) has been used to enhance visualization of malignant tissue during surgery for World Health Organization (WHO) Grade III and IV gliomas. ${ }^{26}$ ALA is a natural biochemical precursor of hemoglobin, which elicits synthesis and accumulation of fluorescent porphyrins in various epithelia and cancerous tissue. ${ }^{19}$ This is very different than the use of fluorescein, which travels through the plasma and reaches the tumor through the disrupted blood-brain barrier. ${ }^{23}$ ALA has been shown in experimental and clinical studies to be taken up by malignant glioma cells, where it is converted into fluorescing porphyrins, ${ }^{25,27,28}$ and has been described as an aid to enhance glioma resection. ${ }^{26}$ ALA is a well-tolerated agent and associated with low rates of adverse events; the most common being abnormal results in liver function test (LFT), transient hypotension, and photosensitivity causing a mild rash. ${ }^{4,26}$

The European data on ALA have been very supportive in terms of facilitating greater EOR and improving 6-month progression-free survival and overall survival. ${ }^{26}$ The diagnostic accuracy of combining ALA with neuronavigation improves sensitivity from approximately $50 \%$ to $90 \%$ and specificity from $60 \%$ to $80 \% .^{9,17}$ Multiple studies have shown that the combination of these 2 modalities has improved EOR rates in high-grade glioma surgery. 5,6,8,22 However, it remains unknown whether the intensity of ALA fluorescence correlates with the degree of tumor cellularity. In this single-center Phase II clinical trial, we aimed to determine whether the intensity of ALA fluorescence is associated with degree of histological cellularity. In addition, we secondarily evaluated for ALAassociated adverse events and complications.

\section{Methods}

This is a single-center, single-arm, open-label Phase II clinical trial of ALA fluorescence-guided resection of high-grade gliomas. This study was formally approved by the University of California, San Francisco, Committee of Human Research.

\section{Primary and Secondary Objectives}

The primary aims of the study were to 1) evaluate the effectiveness of using the presence or the absence of ALA fluorescence to identify the presence or absence of tumor cells, and 2) to determine if the ALA fluorescence intensity helps predict the degree of tumor cellularity based on histological evaluation.

Our secondary objective was to determine the safety profile of a single dose of ALA administered preoperatively at a dose of $20 \mathrm{mg} / \mathrm{kg}$ body weight.

\section{Patient Selection}

At a single institution, patients who planned to undergo craniotomy for high-grade glioma resection were prospectively enrolled over a 43-month period, from August 2010 to February 2014. Eligibility and inclusion criteria were 1) presumptive diagnosis of high-grade glioma (WHO Grade III and IV) based on imaging studies or recurrent highgrade gliomas that had previously undergone diagnosis, 2) aged 18-72 years, 3) Karnofsky Performance Scale score of $60 \%$ or greater, 4) normal organ and marrow function, 5) agreement of adequate use of contraception as the effects of ALA to the developing fetus are unknown, and 6) ability to understand and be willing to give informed consent. Normal organ and marrow function was based on preoperative laboratory values: leukocyte count greater than 3000 per $\mu \mathrm{l}$, absolute neutrophil count greater than 1500 per $\mu$, platelet count greater than 100,000 per $\mu$, total bilirubin within normal institutional limits, aspartate aminotransferase and alanine aminotransferase less than 2.5 times the institution upper limits, and creatinine within normal institutional limits or creatinine clearance greater than or equal to $60 \mathrm{ml} / \mathrm{min} / 1.73 \mathrm{~m}^{2}$. The exclusion criteria for this study were patients who report a history of allergy to compounds of similar chemical or biological composition to ALA, personal or family history of porphyria, uncontrolled intercurrent illness, hypotension, or pregnancy. The patients who were excluded from final analysis were patients who had a diagnosis other than high-grade glioma and/or had insufficient samples to allow histological cellular analysis.

\section{Patients}

Table 1 shows the demographics, tumor characteristics, and perioperative outcomes of patients who underwent ALA fluorescence-guided craniotomy for tumor resection. Over the course of 43 months, 72 patients were enrolled in the study, and of those, 59 patients had adequate data for final analysis (Table 1). The mean age of the cohort was 53.3 years. Of the 59 patients, $69.5 \%$ were male and $30.5 \%$ were female. Surgically treated tumors were located in the frontal lobe in $45.8 \%$ of cases, temporal lobe in $28.8 \%$ of cases, parietal lobe in $16.9 \%$ of cases, insular region in $5.1 \%$ of cases, and occipital lobe in $3.4 \%$ of cases. Slightly more tumors were located in the left hemisphere compared with the right hemisphere $(61.0 \%$ vs $39.0 \%$, respectively), and $74.6 \%$ were in locations near presumed functional brain tissue. A little more than half $(52.5 \%)$ of all brain tumors treated in this cohort were recurrent tumors. The histological diagnoses were 79.7\% GBM, 8.5\% 
TABLE 1. Demographics, tumor characteristics, and perioperative outcomes for patients who underwent ALA-guided craniotomy for tumor resection*

\begin{tabular}{|c|c|}
\hline Variable & Value \\
\hline No. of pts & 59 \\
\hline Age in years & 53.3 \\
\hline \multicolumn{2}{|l|}{ Sex } \\
\hline M & $41(69.5)$ \\
\hline $\mathrm{F}$ & $18(30.5)$ \\
\hline \multicolumn{2}{|l|}{ Tumor location } \\
\hline Frontal & $27(45.8)$ \\
\hline Temporal & $17(28.8)$ \\
\hline Parietal & $10(16.9)$ \\
\hline Insular & $3(5.1)$ \\
\hline Occipital & $2(3.4)$ \\
\hline \multicolumn{2}{|l|}{ Side } \\
\hline Lt & $36(61.0)$ \\
\hline Rt & $23(39.0)$ \\
\hline Recurrence & $31(52.5)$ \\
\hline \multicolumn{2}{|l|}{ Pathological diagnosis } \\
\hline GBM Grade IV & $47(79.7)$ \\
\hline Oligoastrocytoma Grade III & $5(8.5)$ \\
\hline Oligodendroglioma Grade III & $5(8.5)$ \\
\hline Astrocytoma Grade III & $1(1.7)$ \\
\hline Ependymoma Grade III & $1(1.7)$ \\
\hline Complications & $5(8.5)$ \\
\hline Seizure & 2 \\
\hline $\mathrm{IH}$ & 1 \\
\hline Mild rash on torso & 1 \\
\hline$A F$ w/ RVR \& PE & 1 \\
\hline
\end{tabular}

$\mathrm{AF}=$ atrial fibrillation; $\mathrm{IH}=$ intraoperative hypotension; $\mathrm{PE}=$ pulmonary embolus; pts = patients; RVR = rapid ventricular response.

* Values are number (\%) unless otherwise indicated.

WHO Grade III oligoastrocytoma, 8.5\% WHO Grade III oligodendroglioma, 1.7\% WHO Grade III astrocytoma, and $1.7 \%$ WHO Grade III ependymoma.

A total of 5 patients experienced at least 1 complication, resulting in an overall complication rate of $6.8 \%$ (Table 1). Two patients experienced a seizure postoperatively. One patient experienced intraoperative hypotension. Another patient had intraoperative hypotension and developed a rash across the torso, which responded to low oral doses of diphenhydramine. One patient experienced postoperative atrial fibrillation with rapid ventricular response and a pulmonary embolus. ALA was presumed to be the culprit for incidences of intraoperative hypotension and rash, while the other complications were unlikely to be secondary to ALA administration. Therefore, the ALA-associated complication rate among the cohort was $3.4 \%$.

\section{ALA Dosing, Administration, and Postoperative Care}

Prior to surgery, a single administration of ALA at a dose of $20 \mathrm{mg} / \mathrm{kg}$ of body weight was given orally. ALA
TABLE 2. Definition of fluorescence and cellularity grading

\begin{tabular}{|c|c|c|}
\hline \multirow[b]{2}{*}{ Grade } & \multicolumn{2}{|c|}{ Evaluator } \\
\hline & $\begin{array}{l}\text { Surgeon (intensity } \\
\text { of fluorescence) }\end{array}$ & $\begin{array}{l}\text { Pathologist } \\
\text { (cell density) }\end{array}$ \\
\hline 0 & No fluorescence & No definitive tumor cells present \\
\hline 1 & Mild brightness (pink) & $\begin{array}{l}\text { Low abundance of infiltrating } \\
\text { tumor cells }\end{array}$ \\
\hline 2 & $\begin{array}{l}\text { Moderate brightness } \\
\quad \text { (orange) }\end{array}$ & $\begin{array}{l}\text { Moderate abundance of infiltrat- } \\
\text { ing tumor cells }\end{array}$ \\
\hline 3 & $\begin{array}{l}\text { Robust brightness (lava- } \\
\text { like orange) }\end{array}$ & $\begin{array}{l}\text { Highly cellular with abundant } \\
\text { tumor cells }\end{array}$ \\
\hline
\end{tabular}

was dissolved in approximately $100 \mathrm{ml}$ of drinking water or juice and given to the patient approximately 3 hours before anesthesia. Precautions were taken to protect the patient from the effects of skin photosensitivity during surgery and during the postoperative period. Patients received instructions on how to protect themselves and minimize exposure to sunlight and/or indoor lighting for up to 72 hours after administration of ALA. ALA was provided by DUSA Pharmaceuticals/Sun Pharma.

\section{Surgical Procedure}

In all patients included in this study, image-guided microsurgical resection of the tumor was undertaken. During the course of tumor resection under light microscopy, the tumor bed was illuminated with blue light via a high-pass optical filter in the microscope's light source. If fluorescence was present, and if safe, small biopsies were taken from those areas of fluorescence as well as from nonfluorescing regions. When possible, the intent was to collect a number of biopsies with a range of fluorescence intensities from each patient (at least 1 biopsy from nonfluorescent tissue and at least 1 biopsy from the most fluorescent area). All specimens were submitted to neuropathology for review.

\section{Grading of Intensity of Fluorescence and Cell Density}

Biopsies of fluorescent and nonfluorescent tissue within the tumor were graded by the surgeon subjectively at the time of biopsy as fluorescence intensities of 0 , no fluorescence; 1, mild brightness (pink); 2, moderate brightness (orange); or 3, robust brightness (lava-like orange) (Table 2 and Fig. 1). Examples can be seen in Figs. 2 and 3. Biopsies were then provided to the pathologist for grading of cellular density, without knowledge of the surgeon's intraoperative fluorescence grade. The tissue biopsies were immediately placed in zinc-formalin for 4-6 hours, dehydrated in a series of graded alcohols, and infiltrated with low-temperature paraffin for histological analysis. Sections were stained with hematoxylin and eosin $(\mathrm{H} \&$ E) and scored by a neuropathologist. Digital images were captured with a microscope (Olympus, Model BX41TF) and digital microscope camera (Olympus, Model DP70).

For each biopsy, the presence of tumor cells was scored based upon review of $\mathrm{H} \& \mathrm{E}$-stained sections as follows: 0 , no definitive tumor cells present; 1 , low abundance of 

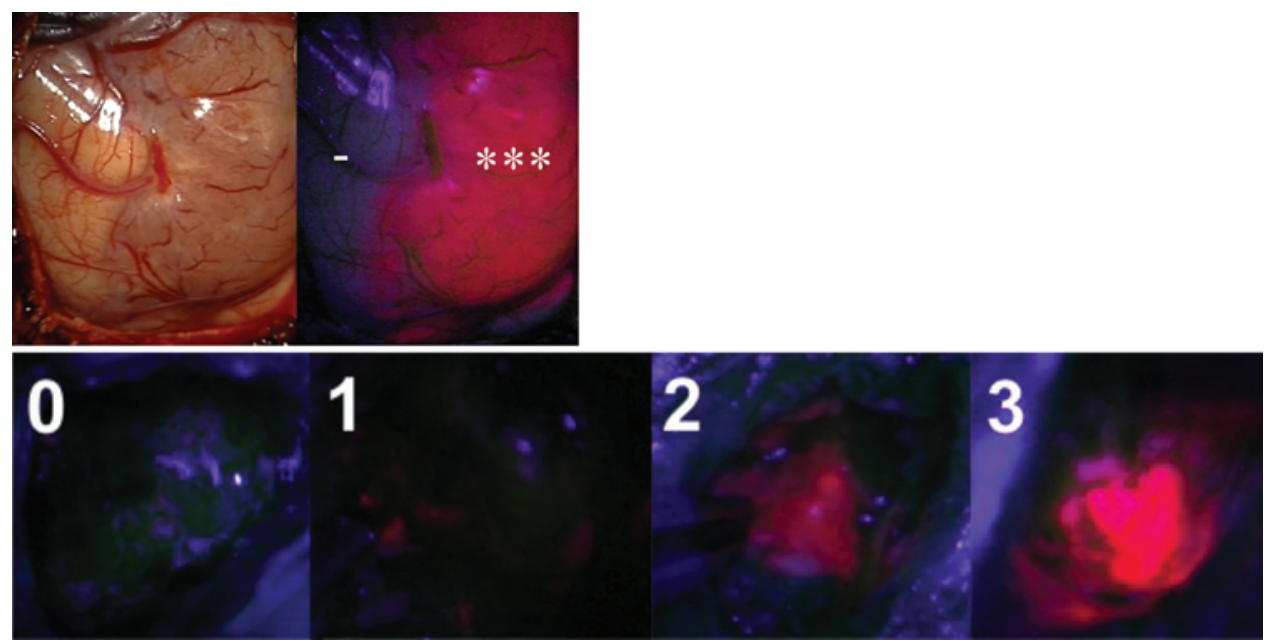

FIG. 1. Representative intraoperative photographs showing grading of ALA fluorescence intensities. Upper: Photograph of normal brain parenchyma and tumor under normal light conditions (left) and illuminated with blue light (right). Nonenhancing tissue is shown in normal brain (-) and a lava-like orange fluorescence is shown in tumor $\left(^{* * *}\right)$. Lower: Photographs showing intraoperative fluorescence intensities 0 through 3 , with $0=$ no fluorescence; 1 = mild brightness, pink; 2 = moderate brightness, orange; and $3=$ robust brightness, lava-like orange. Figure is available in color online only.

infiltrating tumor cells; 2, moderate abundance of infiltrating tumor cells; 3 , highly cellular with abundant tumor cells present (Fig. 4 and Table 2). Tumor cells were identified based upon morphological features including cytological atypia, enlarged nuclear to cytoplasmic volume ratio, and nuclear hyperchromasia. Histopathological evaluation was performed by a semiquantitative analysis of the tissue samples based on a visual estimate of the fraction of tumor nuclei in a sample. This method is similar to the technique used by the clinical pathologist. The pathologist was blinded to ALA fluorescence intensity scores to allow nonbiased cellularity grading. Following the initial analysis of intraoperative and histological scores, the biopsies with a cellularity score of 0 (absence of tumor cells) were reanalyzed and scored for the following features based on evaluation of $\mathrm{H} \& \mathrm{E}$-stained sections: reactive astrocytes, inflammatory cells, necrosis, hypercellular foci, atypical cells, and normal brain parenchyma. Based on this, the biopsies were categorized as either normal brain parenchyma or abnormal brain (presence of reactive astrocytes, inflammatory cells, necrosis, hypercellular regions, or atypical cells).
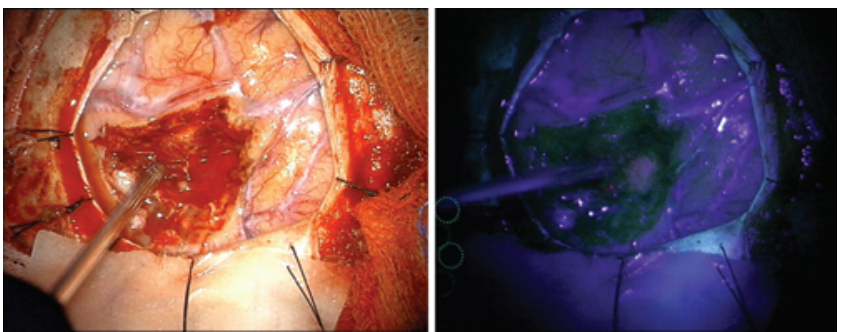

FIG. 2. Intraoperative photographs obtained in a patient with GBM following ALA administration showing the resection cavity under normal light conditions (left) and illuminated with blue light, without evidence of ALA fluorescence (right). Figure is available in color online only.

\section{Adverse Events and Complications}

All patients enrolled were evaluated for signs and symptoms of toxicity. Since potential adverse events related to ALA are known to be transient in nature, it was felt that a 14-day evaluation window was relevant to assess the safety profile of this agent. All complications and adverse events were recorded. The determination of whether a complication or adverse event was secondary to ALA was based on prior reported side effects of ALA. The most common previously reported transient side effects were skin photosensitivity, nausea, vomiting, abdominal discomfort, and lowering of blood pressure, which can result in lightheadedness.

\section{Additional Patient Data}

Demographics, baseline clinical variables, and tumor characteristics were recorded for all patients. These variables included age (years), sex, tumor location (frontal, temporal, parietal, insular, or, occipital), side (left or right), recurrence, and pathological diagnosis.
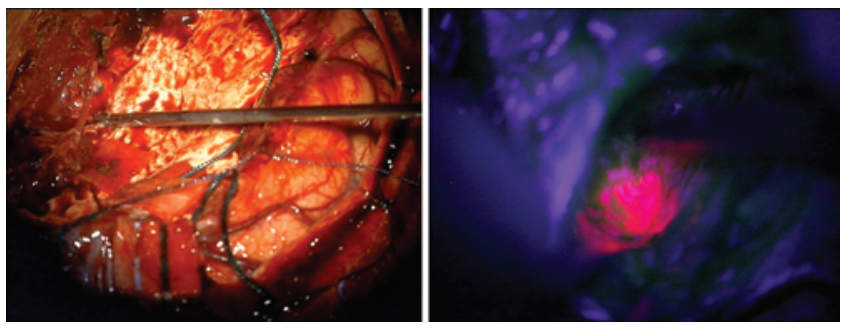

FIG. 3. Intraoperative photographs obtained in a patient with GBM following ALA administration showing the resection cavity under normal light conditions (left) and illuminated with blue light, demonstrating robust lava-like, orange ALA fluorescence (right). Figure is available in color online only. 

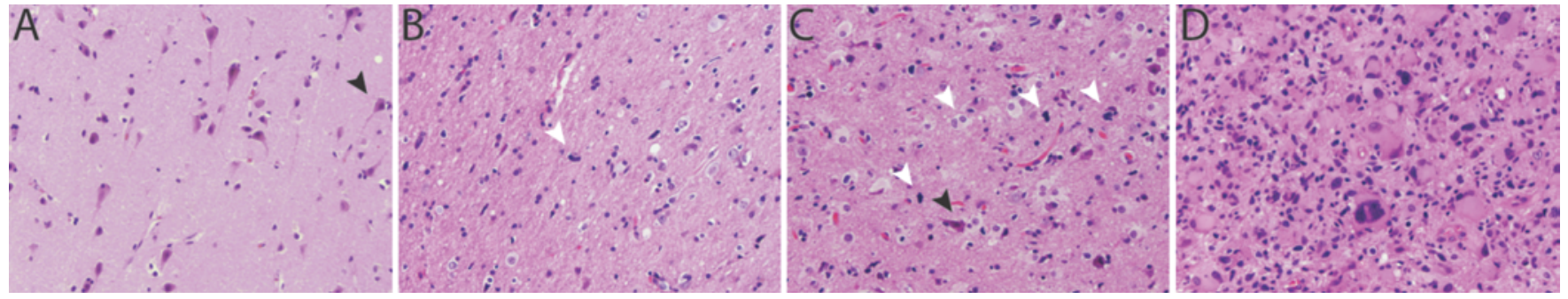

FIG. 4. Representative images of $\mathrm{H} \& \mathrm{E}$-stained sections showing histopathological scoring of tumor biopsies. A: Tumor score 0 showing no definitive neoplastic cells, with relative preservation of cytoarchitecture, including neurons (black arrowhead). B: Tumor score 1 showing low abundance of infiltrating tumor cells (white arrowhead). C: Tumor score 2 showing moderate abundance of tumor cellularity with increased infiltration of tumor cells (white arrowheads) within visible brain cytoarchitecture, such as neurons (black arrowhead). D: Tumor score 3 appearing highly cellular, with abundant tumor cells and no preserved normal brain cytoarchitecture. Original magnification $\times 200$. Figure is available in color online only.

\section{Analysis}

Descriptive statistics were used to define the patient cohort. The cohort was also subcategorized into 3 additional groups: GBM, WHO Grade III gliomas, and recurrent tumors. The frequency with which ALA fluorescence grade matched with cellularity grade was calculated and reported for each tumor type. Overall positive predictive value (PPV) and negative predictive value (NPV) were calculated based on a single biopsy from each patient. The overall PPV was defined as the percentage of all ALA intensity 3 biopsies that have tumorous content: (ALA intensity 3 biopsies that have tumor)/(all ALA intensity 3 biopsies). The overall NPV was defined as the percentage of ALA intensity 0 biopsies that have no tumor content: (ALA intensity 0 biopsies without tumor)/(all ALA intensity 0 biopsies). Next, the Spearman's rank correlation coefficient was calculated to assess the correlation between fluorescent intensity as scored by the neurosurgeon and tumor cellularity as scored by the neuropathologist. Sensitivity, specificity, PPV, and NPV were calculated and reported with confidence intervals. All statistical analysis was performed with SAS 9.3, and 0.05 was used as the threshold for statistical significance.

\section{Results}

\section{ALA Fluorescence and Histological Cellularity}

Table 3 shows the distribution of ALA fluorescence intensity and tumor cellularity grade, stratified by tumor types: all tumors, GBM, Grade III gliomas, and recurrent tumors. A total of 211 biopsies from 59 patients were obtained and graded. There were 175 GBM biopsies, $36 \mathrm{WHO}$ Grade III glioma biopsies, and 110 recurrent tumor biopsies. There were no significant differences in the distribution of intraoperative ALA fluorescence intensity among the different tumor groups $(\mathrm{p}=0.747)$. The distribution of tumor cellularity grade did not demonstrate a significant difference between the 4 groups as well $(p=0.598)$. The overall PPV of utilizing ALA intensity 3 grade as a predictor of tumor presence was high for all 4 groups: all tumor types (97.4\%), GBM (97.2\%), high grade (100.0\%), and recurrent (93.8\%). The NPV of utilizing ALA intensity 0 as a predictor of tumor absence was less impressive: all (37.7\%), GBM (43.9\%), high grade (16.7\%), and recurrent (31.0\%).
Table 4 shows the frequency in which fluorescence intensity and cellularity grade matched. The overall rate at which ALA fluorescence intensity and cellularity grade matched was $32.7 \%$ in all tumor biopsies, 34.9\% in GBM biopsies, $22.2 \%$ in Grade III glioma biopsies, and $29.1 \%$ in recurrent tumor biopsies; there was no significant difference in match rates among the 4 groups $(\mathrm{p}=0.438)$. When fluorescence intensity-cellularity grade match was stratified by particular score, there was no significant difference in the match rate for intensity $0-$ Grade $0(p=0.331)$, intensity $1-$ Grade $1(\mathrm{p}=0.855)$, intensity $2-$ Grade $2(\mathrm{p}=0.971)$, and intensity $3-$ Grade $3(\mathrm{p}=0.951)$ among the 4 groups. Intensity 3-cellularity 3 match rates were particularly high when compared with other intensity-cellularity grades in all groups: all tumors (86.8\%), GBM (86.1\%), Grade III glioma (100.0\%), and recurrent tumors $(87.5 \%)$. In other words, intraoperative ALA fluorescence intensity 3 correlated well with tumor cellularity Grade 3 regardless of tumor type. The Spearman correlation coefficient (r) demonstrated that ALA fluorescence intensity and cellularity grade had strong positive correlations (particularly for intensity 3-cellularity 3$)$ for all groups: all tumors $(\mathrm{k}=0.65, \mathrm{p}$ $<0.001)$, GBM (k = 0.66, $\mathrm{p}<0.001)$, Grade III gliomas $(\mathrm{k}=$ $0.65, \mathrm{p}=0.011)$, and recurrent tumors $(\mathrm{k}=0.62, \mathrm{p}<0.001)$.

\section{Sensitivity and Specificity of ALA Fluorescence}

Table 5 shows the calculated sensitivity, specificity, PPV, and NPV of using ALA fluorescence intensity as a correlative measure for histological cellularity grade. When analyzed by all tumor types, the sensitivity and specificity for ALA to detect any degree of tumor cells on histology were $81.7 \%$ and $64.5 \%$, respectively. PPV was $93.0 \%$ and NPV was $37.7 \%$. Using ALA fluorescence intensity to determine specific cellularity grade was less sensitive but more specific (Table 5). In particular, the specificity and PPV were very high (95.7\% and $86.8 \%$, respectively) for ALA intensity 3-cellularity Grade 3 match. NPV was highest (93.0\%) for ALA intensity 0-cellularity Grade 0 match.

Similar trends in sensitivity, specificity, PPV, and NPV were also seen for samples from GBM and recurrent tumors. For GBM, the overall sensitivity and PPV of detecting any degree of tumor were $84.2 \%$ and $91.8 \%$, respectively. Similarly, in the recurrent tumors, overall 
TABLE 3. ALA fluorescence intensity and histological cellularity grade stratified by tumor type*

\begin{tabular}{|c|c|c|c|c|c|}
\hline Sample & All $(n=211)$ & GBM $(n=175)$ & WHO Grade III $(n=36)$ & Recurrent $(n=110)$ & p Value \\
\hline ALA fluorescence intensity & & & & & 0.747 \\
\hline 0 & $53(25.1)$ & $41(23.4)$ & $12(33.3)$ & $29(26.4)$ & \\
\hline 1 & $60(28.4)$ & $49(28.0)$ & $11(30.6)$ & $33(30.0)$ & \\
\hline 2 & $60(28.4)$ & $49(28.0)$ & $11(30.6)$ & $32(29.1)$ & \\
\hline 3 & $38(18.0)$ & $36(20.6)$ & $2(5.6)$ & $16(14.6)$ & \\
\hline Histological cellularity grade & & & & & 0.598 \\
\hline 0 & $31(14.7)$ & $29(16.6)$ & $2(5.6)$ & $15(13.6)$ & \\
\hline 1 & $33(15.6)$ & $27(15.4)$ & $6(16.7)$ & $23(20.9)$ & \\
\hline 2 & $51(24.2)$ & $38(21.7)$ & $13(36.1)$ & $28(25.5)$ & \\
\hline 3 & $96(45.5)$ & $81(46.3)$ & $15(41.7)$ & $44(40.0)$ & \\
\hline PPV: intensity 3 , presence of tumor $(\%) \dagger$ & $97.4(84.5-99.8)$ & $97.2(83.8-99.9)$ & $100.0(70.0-100.0)$ & $93.8(67.7-99.7)$ & $\mathrm{N} / \mathrm{A}$ \\
\hline NPV: intensity 0 , absence of tumor (\%) $\ddagger$ & $37.7(25.1-52.1)$ & $43.9(28.9-60.1)$ & $16.7(2.9-49.1)$ & $31.0(16.0-51.0)$ & $\mathrm{N} / \mathrm{A}$ \\
\hline
\end{tabular}

$\mathrm{N} / \mathrm{A}=$ not available.

* Values are number (\%) unless otherwise indicated.

$\dagger$ Defined as the percentage of all biopsies taken from the most fluorescent tissues (ALA intensity 3 ) that have tumorous content.

$\ddagger$ Defined as the percentage of all biopsies taken from nonfluorescent tissues (ALA intensity 0 ) with no tumor.

sensitivity was $78.9 \%$, and PPV was $92.6 \%$. When ALA fluorescence intensity was used as a predictor for cellularity grade, sensitivity decreased and specificity increased. Particularly, the specificity of ALA intensity 3-cellularity Grade 3 match was $94.7 \%$ in GBM samples and $97.0 \%$ for recurrent tumors samples; the PPV was $86.1 \%$ in GBM and $87.5 \%$ in recurrent tumors. The NPV for ALA intensity 0 -cellularity Grade 0 matches in GBM was $91.8 \%$ and in recurrent tumors was $92.6 \%$.

Samples from high-grade gliomas demonstrated a slightly different trend of performance measures when using ALA fluorescence intensity as a method to predict cellularity grade (Table 5). Overall sensitivity at detecting the presence of tumor cells with ALA fluorescence was low, at 29.4\%, and NPV was 7.7\%. However, specificity and PPV were both $100.0 \%$. The sensitivity and NPV of ALA intensity 0 -cellularity Grade 0 match were both $100.0 \%$. The specificity and PPV of ALA intensity 3-cellularity Grade 3 match were both $100.0 \%$ as well.

\section{Absence of Fluorescence and Cellularity}

Table 6 shows the distribution of cellularity grade in bi- opsies with no fluorescence (ALA intensity 0 ) and the distribution of ALA intensity grade in biopsies with no tumor cells (cellularity Grade 0). Among biopsies with no tumor cells (cellularity Grade 0 ), $48.4 \%$ contained normal brain tissue and $51.6 \%$ of biopsies contained areas of abnormal (inflammatory) brain parenchyma. Of the biopsies with cellularity Grade $0,35.4 \%$ still demonstrated ALA fluorescence: ALA intensity 1 (29.0\%), ALA intensity 2 (3.2\%), and ALA intensity 3 (3.2\%), and of those biopsies, $90.9 \%$ revealed abnormal brain tissue and $8.1 \%$ had normal brain. In nonfluorescent (ALA intensity 0) biopsies, 62.3\% had tumor cells present: cellularity Grade $1(35.9 \%)$, cellularity Grade 2 (18.9\%), and cellularity Grade 3 (7.6\%).

\section{Discussion}

In this Phase II prospective clinical trial, we evaluated whether ALA fluorescence intensity was able to distinguish degrees of tumor cellularity on histological analysis. Overall, ALA intensity 3 demonstrated high PPV for the presence of tumor, and ALA intensity 0 demonstrated low NPV for absence of tumor. ALA fluorescence intensities

TABLE 4. ALA fluorescence-cellularity match rate stratified by tumor type*

\begin{tabular}{lccccc}
\hline \multicolumn{1}{c}{ Sample } & All & GBM & WHO Grade III & Recurrent & p Value \\
\hline ALA fluorescence-cellularity match (\%) & & & & & \\
\hline All grades & 32.7 & 34.9 & 22.2 & 29.1 & 0.438 \\
\hline Intensity 0-Grade 0 & 37.7 & 43.9 & 16.7 & 31.0 & 0.331 \\
\hline Intensity 1-Grade 1 & 11.7 & 10.2 & 18.8 & 15.2 & 0.855 \\
\hline Intensity 2-Grade 2 & 15.0 & 14.3 & 18.8 & 12.5 & 0.971 \\
\hline Intensity 3-Grade 3 & 86.8 & 86.1 & 100.0 & 87.5 & 0.951 \\
\hline Spearman correlation coefficient $(r)$ & 0.65 & 0.66 & 0.65 & 0.62 & $\mathrm{~N} / \mathrm{A}$ \\
\hline p value & $<0.001$ & $<0.001$ & 0.011 & $<0.001$ & $\mathrm{~N} / \mathrm{A}$ \\
\hline
\end{tabular}

* Values are \% frequency. 
TABLE 5. Performance measures of ALA fluorescence in determining specific tumor cellularity*

\begin{tabular}{|c|c|c|c|c|}
\hline ALA Intensity-Cellularity Grade & Sensitivity & Specificity & PPV & NPV \\
\hline \multicolumn{5}{|l|}{ All tumors $(n)$} \\
\hline Presence of fluoresce-presence of tumor & $81.7(75.1-86.9)$ & $64.5(45.4-80.1)$ & $93.0(87.6-96.3)$ & $37.7(25.1-52.1)$ \\
\hline $0-0$ & $64.5(45.3-80.2)$ & $81.7(75.1-86.9)$ & $37.7(25.1-52.1)$ & $93.0(87.6-96.3)$ \\
\hline $1-1$ & $21.2(9.6-39.4)$ & $70.2(62.8-76.7)$ & $11.7(5.2-23.2)$ & $82.8(75.6-88.2)$ \\
\hline $2-2$ & $17.6(8.9-31.4)$ & $68.1(60.2-75.1)$ & $15.0(7.5-27.1)$ & $72.2(64.2-79.0)$ \\
\hline $3-3$ & $34.4(35.1-44.8)$ & $95.7(89.7-98.3))$ & $86.8(71.1-95.1)$ & $63.6(55.9-70.7)$ \\
\hline \multicolumn{5}{|l|}{ GBM } \\
\hline Presence of fluoresce-presence of tumor & $84.2(76.9-88.5)$ & $62.1(42.4-78.7)$ & $91.8(85.4-95.6)$ & $43.9(28.8-60.1)$ \\
\hline $0-0$ & $62.1(42.4-78.7)$ & $84.2(77.1-89.6)$ & $43.9(28.9-60.1)$ & $91.8(85.4-95.6)$ \\
\hline $1-1$ & $18.5(7.0-38.7)$ & $70.3(62.1-77.4)$ & $10.2(3.8-23.0)$ & $82.5(74.5-88.5)$ \\
\hline $2-2$ & $18.4(8.3-34.9)$ & $69.3(60.8-76.8)$ & $14.3(6.4-27.9)$ & $75.4(66.8-82.4)$ \\
\hline $3-3$ & $38.3(27.9-49.8)$ & $94.7(87.5-98.0)$ & $86.1(69.7-94.8)$ & $64.0(55.4-71.9)$ \\
\hline \multicolumn{5}{|l|}{ WHO Grade III } \\
\hline Presence of fluoresce-presence of tumor & $29.4(14.8-45.4)$ & $100.0(19.8-100.0)$ & $100.0(65.5-100.0)$ & $7.7(1.3-26.6)$ \\
\hline $0-0$ & $100.0(19.8-100.0)$ & $70.6(52.3-84.3)$ & $16.7(2.9-49.1)$ & $100.0(82.8-100.0)$ \\
\hline $1-1$ & $33.3(6.0-75.9)$ & $70.0(50.4-84.6)$ & $18.2(3.2-52.2)$ & $84.0(63.1-94.7)$ \\
\hline $2-2$ & $15.4(2.7-46.3)$ & $60.9(38.8-79.5)$ & $18.2(3.2-52.2)$ & $56.0(35.3-75.0)$ \\
\hline $3-3$ & $11.8(2.1-37.7)$ & $100.0(79.1-100.0)$ & $100.0(19.8-100.0)$ & $55.9(38.1-72.4)$ \\
\hline \multicolumn{5}{|l|}{ Recurrence } \\
\hline Presence of fluoresce-presence of tumor & $78.9(69.1-86.4)$ & $60.0(32.9-82.5)$ & $92.6(84.0-97.0)$ & $31.0(16.0-51.0)$ \\
\hline $0-0$ & $60.0(32.9-82.5)$ & $78.9(69.1-86.4)$ & $31.0(16.0-51.0)$ & $92.6(84.0-97.0)$ \\
\hline $1-1$ & $21.7(8.3-44.2)$ & $67.8(56.8-77.2)$ & $15.2(5.7-32.7)$ & $76.6(65.3-85.2)$ \\
\hline $2-2$ & $14.3(4.7-33.6)$ & $65.9(54.5-75.7)$ & $12.5(4.1-29.9)$ & $69.2(57.6-78.9)$ \\
\hline $3-3$ & $31.8(19.1-47.7)$ & $97.0(88.5-99.4)$ & $87.5(60.4-97.8)$ & $68.1(57.6-77.1)$ \\
\hline
\end{tabular}

${ }^{*}$ Values are $\%(95 \% \mathrm{Cl})$.

of 1 and 2 did not readily correlate with specific tumor cellular densities; only ALA fluorescence intensity 3 correlated strongly with tumor cellularity Grade 3 (more than $85 \%$ of the time). In addition, this study was able to show that ALA fluorescence continues to be present in $35.4 \%$ of samples that did not contain tumor cells, and $62.3 \%$ of tumor infiltrated tissue did not display ALA fluorescence. Among the cohort, ALA-associated adverse events and complications were uncommon and transient. Therefore, the benefit ratio of utilizing ALA as a guide to high-grade glioma surgery likely outweighs its risk and should be considered as a routine surgical adjunct in this setting. In general, ALA has proven to be a very useful tool to enhance the resection of high-grade gliomas and is a good marker of tumor cellularity, especially in brightly fluorescent areas (i.e., lava-like orange).

The primary outcome of interest was the PPV of utilizing the most fluorescent grade as a predictor for tumor presence, and in this respect, ALA was able to do so $97.4 \%$ of the time (i.e., this means in the presence of strong fluorescence there is a very high probability that tumor is present). This high PPV is consistent with a prior study, finding that strong fluorescence as a test for the presence of tumor cells had a PPV of $100 \% .{ }^{34}$ Conversely, in the absence of ALA fluorescence, the probability of having absence of tumor was $37.7 \%$ (NPV) (i.e., this demonstrates that the absence of ALA fluorescence does not predictably indicate the absence of tumor). Both of these findings have significant clinical implications as this may influence surgical decision making when confronted with areas of high ALA fluorescence and the absence of ALA fluorescence.

As of every test, ALA fluorescence is not always able to distinguish or predict the presence of absence of tumor cells. ALA has been described to be selectively taken up by glioma cells..$^{25,27,28}$ However, the findings from this study suggest that ALA fluorescence does not have perfect correlation with tumor cell identification. ${ }^{20}$ In samples in which no fluorescence was detected, more than half (62.3\%) of those samples had tumor cells present on his-

\section{TABLE 6. Absence of fluorescence and tumor cellularity}

\begin{tabular}{ccccl}
\hline Characteristic & \multicolumn{4}{c}{ Intensity or Grade } \\
\hline Fluorescence intensity & 0 & 1 & 2 & 3 \\
\hline No tumor cellularity $(\%)$ & 64.5 & $29.0^{*}$ & $3.2^{*}$ & $3.2^{*}$ \\
\hline Tumor cellularity grade & 0 & 1 & 2 & 3 \\
\hline No fluorescence $(\%)$ & 37.7 & 35.9 & 18.9 & 7.6 \\
\hline
\end{tabular}

* Of the biopsies that had fluorescence but no tumor, $90.9 \%$ had abnormal brain tissue characterized by reactive astrocytes and scattered inflammatory cells, and $8.1 \%$ had normal brain. 
tology. Similarly, a prior study showed that $73.7 \%$ of nonfluorescent samples still contained tumor. ${ }^{20}$ These findings suggest that there may be particular tumor cells that do not consistently take up or metabolize ALA in a manner that results in visible fluorescence. However, the absence of macroscopic ALA fluorescence does not definitely indicate the absence of ALA or fluorescent porphyrins. A study has shown that spectrometric fluorescence can be detected in marginal tissue without macroscopic fluorescence. ${ }^{29}$ Another possibility is the inability of the microscope's light source to reach deep resection corridors, thus limiting the assessment for adequate ALA fluorescence. Scheduling and optimal dosing of ALA administration prior to surgery should also be considered for ALA uptake into tumor cells. In this study, a 1-time oral dose of ALA was administered approximately 3 hours prior to anesthesia. Unfortunately, the exact time period in which ALA was administered to the time of collection of biopsy samples was not recorded; therefore, the correlation between ALA fluorescence intensity with operative time cannot be determined. The timing of ALA administration should be taken into account in future studies of ALA.

Conversely, $35.4 \%$ of samples had no definitive tumor cells present but continued to demonstrate ALA fluorescence. Post hoc histological examination of these particular samples revealed that almost all samples contained abnormal brain tissue. This finding is similar to a prior study, which showed that approximately $17 \%$ of GBM samples with ALA fluorescence showed no tumor cells but contained areas of peritumoral edema, inflammatory cells, and reactive astrocyte infiltration. ${ }^{30}$ It is also suggested that inflammatory cells such as macrophages may be able to accumulate and convert ALA to its associated fluorescent porphyrins. ${ }^{7}$ This false-positive phenomenon has been described in ALA-guided resection of cerebral metastasis. ${ }^{11,30}$ Interestingly, there was 1 biopsy among our study cohort that demonstrated ALA fluorescence in the presence of completely normal brain tissue. While additional samples are needed to support this finding, this may suggest that even in the absence of tumor, surrounding brain may take up ALA and fluoresce. Alternatively, as the tissue biopsy was not serially sectioned, it is possible that small portions of tumor could be absent from the representative section analyzed by histology.

Previous studies have shown that ALA-guided resection of malignant gliomas have a diagnostic sensitivity of $75 \%$ to $87 \%$ when correlating the presence of ALA fluorescence and the presence of tumor on histological analysis. ${ }^{20,37} \mathrm{~A}$ similar sensitivity of $81.7 \%$ was seen in our study when all tumor types were assessed as a single cohort. However, subgroup analysis of WHO Grade III gliomas had an extremely low sensitivity of $29.4 \%$. This meant the probability of detecting tumor cells on histology with ALA fluorescence occurred only $29.4 \%$ of the time, i.e., ALA fluorescence was a poor indicator to rule out the presence of tumor for WHO Grade III gliomas. Conversely, in WHO Grade III gliomas, specificity was $100.0 \%$, meaning that in the presence of ALA fluorescence there were tumor cells present $100 \%$ of the time, i.e., ALA fluorescence was a good diagnostic indicator to rule in the presence of tumor cells for WHO Grade III gliomas. The specificities ob- served in this study for all tumor types (65\%), GBM (62\%), and recurrent tumors $(60 \%)$ were lower than the previously reported rates of $71 \%$ to $89 \%{ }^{8}$ While ALA fluorescence was observed to have low NPVs, the PPVs were very high with rates greater than $90.0 \%$.

There has been an ongoing interest in determining whether ALA fluorescence is able to specify tumor type, degree of malignancy, and cellularity ${ }^{18}$ Higher levels of fluorescence have higher levels of ALA-induced protoporphyrin concentration and correlate strongly with increasing malignancy (histopathological score) and tissue proliferation. ${ }^{2,31}$ To our knowledge, there are only 2 studies that have directly addressed the relationship of intraoperative ALA fluorescence intensity with degree of cellularity. ${ }^{20,29}$ The first study by Roberts et al. included a total of 124 biopsy specimens from 11 patients with GBM. ${ }^{20}$ The authors graded intraoperative ALA fluorescence and cellularity on a 4-point scale. They showed that as histopathological score and tumor burden increased, the proportion of fluorescent samples as well as the level of fluorescence score also increased. More recently Stummer et al. performed a prospective study to assess the reliability of visible fluorescence qualities by spectrometry, pathology, and imaging among 33 patients with malignant gliomas. ${ }^{29}$ Strong ALA fluorescence was associated with greater spectrometric fluorescence, proliferating tumor, and cell density. Weaker ALA fluorescence was associated with less spectrometric fluorescence, proliferating tumor, and cell density.

In comparison with our study, we did not observe a linear trend in correlation between ALA fluorescent intensity and cellularity, but we did find that ALA fluorescence intensity 3 had a very high match rate to cellularity Grade 3. In fact, the Spearman correlation coefficients suggested a strong positive correlation. The specificity and PPV of ALA intensity 3-cellularity Grade 3 was very high, ranging from $95 \%$ to $100 \%$ and $86 \%$ to $100 \%$, respectively. These PPVs are similar to the $100 \%$ PPV seen in the study by Stummer et al. ${ }^{29}$ However, we observed much lower PPVs (less than 45\%) for lower ALA intensities. These findings suggest that the presence of high fluorescence likely correlates with not only the presence of tumor but greater tumor burden.

ALA has been described as a well-tolerated agent and associated with low rates of adverse events. ${ }^{4,26}$ The most common adverse events and complications associated with ALA are abnormal results in LFTs less than 24 hours after surgery, hypotension, and photosensitivity, causing a transient rash. ${ }^{4,26}$ Among our cohort, 1 patient experienced intraoperative hypotension and 1 other patient experienced both hypotension and a mild rash, which were assumed to be associated with ALA administration. This resulted in an ALA-associated complication rate of 3.4\%. A study by Chung et al., performed a comparative analysis to assess the incidence and risk factors contributing to ALAinduced side effects and adverse advents. ${ }^{4}$ Cardiovascular disease and use of hypertensive medications were associated with higher rates of hypotensive adverse events. In the same study, while not statistically significant, a higher proportion of patients in the ALA group had seizures compared with patients without ALA. Overall, oral ALA administration is safe and associated with low rates of minor 
complications. The benefit it offers during glioma surgery outweighs the risk associated with the agent.

There are a few limitations to this study that should be briefly discussed. First, the lack of an objective or quantitative measure for intraoperative ALA fluorescence intensity is a drawback. The lack of an objective measure for fluorescence makes it difficult to assign an intermediate score such as intensity 1 and intensity 2 . Therefore, linearity or correlations among these grades may be lacking secondary to this drawback. Future studies need to formally address this by utilizing specialized equipment that is able to quantify fluorescence. An approach to objectively quantify intraoperative ALA fluorescence intensity is to use a fiberoptic probe. The blunt end of the probe is placed in gentle contact with brain or tumor tissue for 3-5 seconds. This allows spectroscopic measurement of protoporphyrin IX concentration. Then, the absolute concentration of protoporphyrin IX is used to quantify ALA fluorescence with the use of a mathematical model. This technique has been shown to be rather sensitive in quantifying ALA fluorescence intensity, even in areas not readily visualized by routine gross inspection. ${ }^{32}$ Another minor limitation was the small sample size of patients with WHO Grade III gliomas. While, our study was, in fact, designed to study WHO Grade III and IV gliomas, most patients enrolled had a diagnosis of GBM. Therefore, the overall results of this study may be most applicable to GBM. There also needs to be caution regarding the use of PPV and NPV on a cohort of patients that do not represent the normal probability of a disease, because PPV and NPV are dependent on prevalence. The cohort in this study had definitive disease (i.e., they were known to have a diagnosis of brain tumor); thus, the PPV and NPV can be slightly biased estimates. Despite these limitations, the findings from this study have direct clinical applicability.

\section{Conclusions}

The PPV of utilizing most intense ALA fluorescence intensity (lava-like orange) as a predictor of tumor presence is high. However, the NPV of utilizing the absence of fluorescence as an indicator of no tumor is low. ALA intensity is a strong predictor for degree of tumor cellularity for the most fluorescent areas but less so for lower ALA intensities. This study also demonstrates that tumorinfiltrated tissue may not illuminate, and, conversely, areas without tumor cells may exhibit ALA fluorescence. Even in the absence of tumor cells, reactive changes including inflammation may lead to ALA fluorescence. ALA-associated adverse events and complications are uncommon, minor, and transient. ALA should be considered as a viable option for patients undergoing craniotomy for highgrade glioma resection given the extremely low rates of associated complications.

\section{References}

1. Albert FK, Forsting M, Sartor K, Adams HP, Kunze S: Early postoperative magnetic resonance imaging after resection of malignant glioma: objective evaluation of residual tumor and its influence on regrowth and prognosis. Neurosurgery 34:45-61, 1994

2. Arita H, Kinoshita M, Kagawa N, Fujimoto Y, Kishima H,
Hashimoto N, et al: ${ }^{11} \mathrm{C}$-methionine uptake and intraoperative 5-aminolevulinic acid-induced fluorescence as separate index markers of cell density in glioma: a stereotactic imagehistological analysis. Cancer 118:1619-1627, 2012

3. Barker FG II, Prados MD, Chang SM, Gutin PH, Lamborn KR, Larson DA, et al: Radiation response and survival time in patients with glioblastoma multiforme. J Neurosurg 84:442-448, 1996

4. Chung IW, Eljamel S: Risk factors for developing oral 5-aminolevulinic acid-induced side effects in patients undergoing fluorescence guided resection. Photodiagn Photodyn Ther 10:362-367, 2013

5. Della Puppa A, Ciccarino P, Lombardi G, Rolma G, Cecchin D, Rossetto M: 5-Aminolevulinic acid fluorescence in high grade glioma surgery: surgical outcome, intraoperative findings, and fluorescence patterns. BioMed Res Int 2014:232561, 2014

6. Della Puppa A, De Pellegrin S, d'Avella E, Gioffrè G, Rossetto M, Gerardi A, et al: 5-aminolevulinic acid (5-ALA) fluorescence guided surgery of high-grade gliomas in eloquent areas assisted by functional mapping. Our experience and review of the literature. Acta Neurochir (Wien) 155:965-972, 2013

7. Dietze A, Berg K: ALA-induced porphyrin formation and fluorescence in synovitis tissue In-vitro and in vivo studies. Photodiagn Photodyn Ther 2:299-307, 2005

8. Díez Valle R, Tejada Solis S, Idoate Gastearena MA, García de Eulate R, Domínguez Echávarri P, Aristu Mendiroz J: Surgery guided by 5 -aminolevulinic fluorescence in glioblastoma: volumetric analysis of extent of resection in singlecenter experience. J Neurooncol 102:105-113, 2011

9. Hefti M, von Campe G, Moschopulos M, Siegner A, Looser $\mathrm{H}$, Landolt $\mathrm{H}$ : 5-aminolevulinic acid induced protoporphyrin IX fluorescence in high-grade glioma surgery: a one-year experience at a single institution. Swiss Med Wkly 138:180185,2008

10. Ji M, Orringer DA, Freudiger CW, Ramkissoon S, Liu X, Lau D, et al: Rapid, label-free detection of brain tumors with stimulated Raman scattering microscopy. Sci Transl Med 5:201ra119, 2013

11. Kamp MA, Grosser P, Felsberg J, Slotty PJ, Steiger HJ, Reifenberger G, et al: 5-aminolevulinic acid (5-ALA)-induced fluorescence in intracerebral metastases: a retrospective study. Acta Neurochir (Wien) 154:223-228, 2012

12. Lacroix M, Abi-Said D, Fourney DR, Gokaslan ZL, Shi W, DeMonte F, et al: A multivariate analysis of 416 patients with glioblastoma multiforme: prognosis, extent of resection, and survival. J Neurosurg 95:190-198, 2001

13. Lindner D, Trantakis C, Renner C, Arnold S, Schmitgen A, Schneider J, et al: Application of intraoperative 3D ultrasound during navigated tumor resection. Minim Invasive Neurosurg 49:197-202, 2006

14. Marko NF, Weil RJ, Schroeder JL, Lang FF, Suki D, Sawaya RE: Extent of resection of glioblastoma revisited: personalized survival modeling facilitates more accurate survival prediction and supports a maximum-safe-resection approach to surgery. J Clin Oncol 32:774-782, 2014

15. Nimsky C, Ganslandt O, Buchfelder M, Fahlbusch R: Intraoperative visualization for resection of gliomas: the role of functional neuronavigation and intraoperative 1.5 T MRI. Neurol Res 28:482-487, 2006

16. Orringer D, Lau D, Khatri S, Zamora-Berridi GJ, Zhang K, Wu C, et al: Extent of resection in patients with glioblastoma: limiting factors, perception of resectability, and effect on survival. J Neurosurg 117:851-859, 2012

17. Panciani PP, Fontanella M, Garbossa D, Agnoletti A, Ducati A, Lanotte M: 5-aminolevulinic acid and neuronavigation in high-grade glioma surgery: results of a combined approach. Neurocirugia (Astur) 23:23-28, 2012 
18. Piccirillo SG, Dietz S, Madhu B, Griffiths J, Price SJ, Collins $\mathrm{VP}$, et al: Fluorescence-guided surgical sampling of glioblastoma identifies phenotypically distinct tumour-initiating cell populations in the tumour mass and margin. Br $\mathbf{J}$ Cancer 107:462-468, 2012

19. Regula J, MacRobert AJ, Gorchein A, Buonaccorsi GA, Thorpe SM, Spencer GM, et al: Photosensitisation and photodynamic therapy of oesophageal, duodenal, and colorectal tumours using 5 aminolaevulinic acid induced protoporphyrin IX-a pilot study. Gut 36:67-75, 1995

20. Roberts DW, Valdés PA, Harris BT, Fontaine KM, Hartov A, Fan X, et al: Coregistered fluorescence-enhanced tumor resection of malignant glioma: relationships between $\delta$ aminolevulinic acid-induced protoporphyrin IX fluorescence, magnetic resonance imaging enhancement, and neuropathological parameters. Clinical article. J Neurosurg 114:595603, 2011

21. Sanai N, Polley MY, McDermott MW, Parsa AT, Berger MS: An extent of resection threshold for newly diagnosed glioblastomas. J Neurosurg 115:3-8, 2011

22. Schucht P, Beck J, Abu-Isa J, Andereggen L, Murek M, Seidel $\mathrm{K}$, et al: Gross total resection rates in contemporary glioblastoma surgery: results of an institutional protocol combining 5-aminolevulinic acid intraoperative fluorescence imaging and brain mapping. Neurosurgery 71:927-936, 2012

23. Shinoda J, Yano H, Yoshimura S, Okumura A, Kaku Y, Iwama T, et al: Fluorescence-guided resection of glioblastoma multiforme by using high-dose fluorescein sodium. Technical note. J Neurosurg 99:597-603, 2003

24. Simpson JR, Horton J, Scott C, Curran WJ, Rubin P, Fischbach J, et al: Influence of location and extent of surgical resection on survival of patients with glioblastoma multiforme: results of three consecutive Radiation Therapy Oncology Group (RTOG) clinical trials. Int J Radiat Oncol Biol Phys 26:239-244, 1993

25. Stummer W, Novotny A, Stepp H, Goetz C, Bise K, Reulen HJ: Fluorescence-guided resection of glioblastoma multiforme by using 5-aminolevulinic acid-induced porphyrins: a prospective study in 52 consecutive patients. J Neurosurg 93:1003-1013, 2000

26. Stummer W, Pichlmeier U, Meinel T, Wiestler OD, Zanella F, Reulen HJ: Fluorescence-guided surgery with 5-aminolevulinic acid for resection of malignant glioma: a randomised controlled multicentre phase III trial. Lancet Oncol 7:392401, 2006

27. Stummer W, Stocker S, Novotny A, Heimann A, Sauer O, Kempski O, et al: In vitro and in vivo porphyrin accumulation by C6 glioma cells after exposure to 5-aminolevulinic acid. J Photochem Photobiol B 45:160-169, 1998

28. Stummer W, Stocker S, Wagner S, Stepp H, Fritsch C, Goetz $\mathrm{C}$, et al: Intraoperative detection of malignant gliomas by 5 -aminolevulinic acid-induced porphyrin fluorescence. Neurosurgery 42:518-526, 1998

29. Stummer W, Tonn JC, Goetz C, Ullrich W, Stepp H, Bink A, et al: 5-Aminolevulinic acid-derived tumor fluorescence: the diagnostic accuracy of visible fluorescence qualities as corroborated by spectrometry and histology and postoperative imaging. Neurosurgery 74:310-320, 2014

30. Utsuki S, Oka H, Sato S, Shimizu S, Suzuki S, Tanizaki Y, et al: Histological examination of false positive tissue resection using 5-aminolevulinic acid-induced fluorescence guidance. Neurol Med Chir (Tokyo) 47:210-214, 2007

31. Valdés PA, Kim A, Brantsch M, Niu C, Moses ZB, Tosteson $\mathrm{TD}$, et al: $\delta$-aminolevulinic acid-induced protoporphyrin IX concentration correlates with histopathologic markers of malignancy in human gliomas: the need for quantitative fluorescence-guided resection to identify regions of increasing malignancy. Neuro Oncol 13:846-856, 2011

32. Valdés PA, Leblond F, Kim A, Harris BT, Wilson BC, Fan $X$, et al: Quantitative fluorescence in intracranial tumor: implications for ALA-induced PpIX as an intraoperative biomarker. J Neurosurg 115:11-17, 2011

33. Vecht CJ, Avezaat CJ, van Putten WL, Eijkenboom WM, Stefanko SZ: The influence of the extent of surgery on the neurological function and survival in malignant glioma. A retrospective analysis in 243 patients. J Neurol Neurosurg Psychiatry 53:466-471, 1990

34. Widhalm G, Minchev G, Woehrer A, Preusser M, Kiesel B, Furtner J, et al: Strong 5-aminolevulinic acid-induced fluorescence is a novel intraoperative marker for representative tissue samples in stereotactic brain tumor biopsies. Neurosurg Rev 35:381-391, 2012

35. Willems PW, Taphoorn MJ, Burger H, Berkelbach van der Sprenkel JW, Tulleken CA: Effectiveness of neuronavigation in resecting solitary intracerebral contrast-enhancing tumors: a randomized controlled trial. J Neurosurg 104:360-368, 2006

36. Wirtz CR, Albert FK, Schwaderer M, Heuer C, Staubert A, Tronnier VM, et al: The benefit of neuronavigation for neurosurgery analyzed by its impact on glioblastoma surgery. Neurol Res 22:354-360, 2000

37. Zhao S, Wu J, Wang C, Liu H, Dong X, Shi C, et al: Intraoperative fluorescence-guided resection of high-grade malignant gliomas using 5-aminolevulinic acid-induced porphyrins: a systematic review and meta-analysis of prospective studies. PLoS One 8:e63682, 2013

\section{Disclosure}

The authors report no conflict of interest concerning the materials or methods used in this study or the findings specified in this paper.

\section{Author Contributions}

Conception and design: Berger, Chang, McDermott, Phillips. Acquisition of data: Berger, McDermott, Phillips. Analysis and interpretation of data: Berger, Lau, Hervey-Jumper, Chang, Molinaro, Phillips. Drafting the article: Lau, Hervey-Jumper. Critically revising the article: Berger, Lau, Hervey-Jumper, Chang, McDermott, Phillips. Reviewed submitted version of manuscript: all authors. Approved the final version of the manuscript on behalf of all authors: Berger. Statistical analysis: Lau, Molinaro. Study supervision: Berger.

\section{Correspondence}

Mitchel S. Berger, Chair Department of Neurological Surgery, University of California, San Francisco, 505 Parnassus Ave., M779, San Francisco, California 94143. email: bergerm@ neurosurg.ucsf.edu. 